In der Rubrik „Literatur kompakt" werden die wichtigsten Originalarbeiten aus der internationalen Fachliteratur referiert.

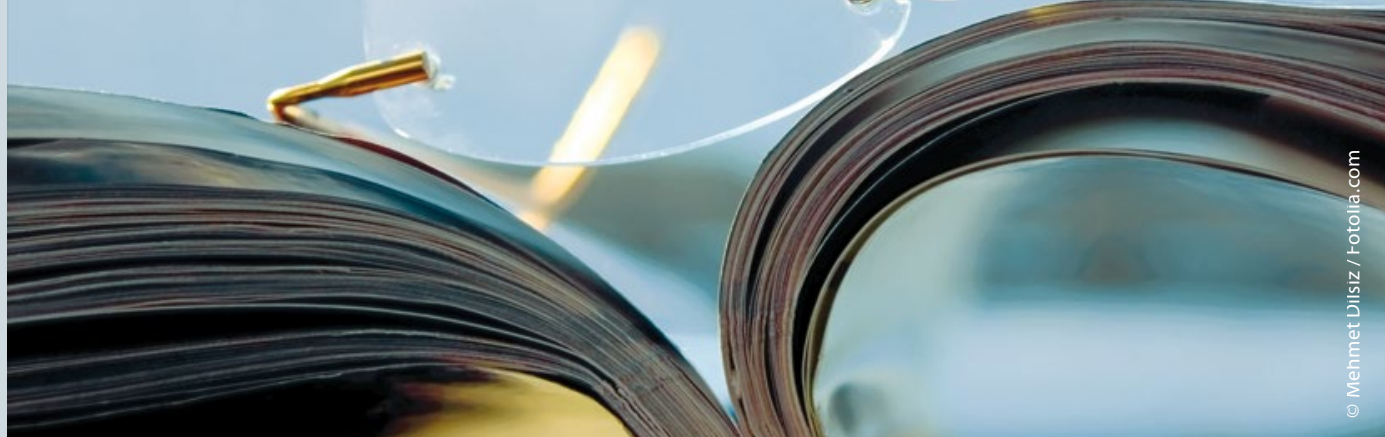

\section{Diabetische Neuropathie: Topisches Capsaicin reduziert den Schmerz}

\section{Capsaicin-Pflaster sind eine bewährte Option in der analgetischen Lokal- therapie bei Neuralgien und Polyneuropathien. Nun liegt erstmals eine Studie zum Effekt bei der schmerzhaften diabetischen Polyneuropathie vor.}

S chmerzhafte Polyneuropathien bei Diabetikern werden meist systemisch mit Antidepressiva, Antikonvulsiva oder Opioiden therapiert. Dosistitrationen, Neben- und Wechselwirkungen sowie das Abhängigkeitspotenzial limitieren den Einsatz dieser Pharmaka bei gleichzeitig nicht sehr hoher Evidenz. In der Zweitlinie werden $8 \%$-Capasaicin-Pflaster empfohlen. Lokales Capsaicin hemmt die hyperaktiven Neuronen, wird kaum systemisch resorbiert und muss nicht titriert werden. Studien bei postherpetischen Neuralgien und HIV-assoziierten Polyneuropathien attestierten dem Paprikascharfstoff eine über 12 Wochen anhaltende Schmerzreduktion nach nur einer rund 30-minütigen Applikation. In der STEP-Studie wurden nun erstmalig Wirksamkeit und Sicherheit bei der diabetischen Polyneuropathie überprüft.

Insgesamt 369 Patienten mit einer diabetischen Polyneuropathie erhielten randomisiert über 30 Minuten entweder das 8\%-Capsaicin- oder ein Placebopflaster direkt auf die schmerzenden Stellen auf dem Fußrücken appliziert. Um die Verblindung aufrechtzuerhalten, fanden Behandlung und Auswertung durch zwei getrennte Ärztegruppen statt und die Patienten erhielten die Information, dass das typische Brennen auch unter Placebo auftreten könne.
Beim primären Studienendpunkt, dem durchschnittlichen Rückgang des „Numeric Pain Rating Scale Scores“ zwischen Studienbeginn und den Wochen 2 bis 8, war das Capsaicin-Pflaster Placebo moderat, aber statistisch signifikant überlegen ( $-27,4$ vs. $-20,9 \%$; $=0,25$ ). Der Wirkunterschied wurde ab Woche 2 sichtbar und blieb bis zur Woche 12 erhalten. Auch Schlafstörungen besserten sich unter Verum im Vergleich zu Placebo in moderatem Ausmaß.

Rund ein Drittel der Patienten der Capsaicin-Gruppe berichteten über passagere Lokalreaktionen wie Schmerzen und Brennen, die teilweise mit Akutanalgetika behandelt werden mussten. Ansonsten bestanden zwischen beiden Gruppen keine Unterschiede im Nebenwirkungsprofil. Die sensorische Wahrnehmung zum Beispiel von Wärme, Kälte und Vibrationsreizen veränderte sich unter Capsaicin ebenfalls nicht.

Fazit: Bei Patienten mit diabetischer Polyneuropathie reduziert die einmalige 30-minütige Applikation eines 8\%-Capsaicin-Pflasters die neuropathischen Schmerzen im Vergleich zu Placebo moderat, statistisch signifikant und lang anhaltend über bis zu 12 Wochen. Der Wirkeffekt ist vergleichbar mit dem anderer Therapieoptionen, die Verträglichkeit gut.

Dr. Barbara Kreutzkamp

Simpson DM et al. Capsaicin $8 \%$ patch in painful diabetic peripheral neuropathy: a randomized, double-blind, placebo-controlled study. J Pain 2017;18:42-53

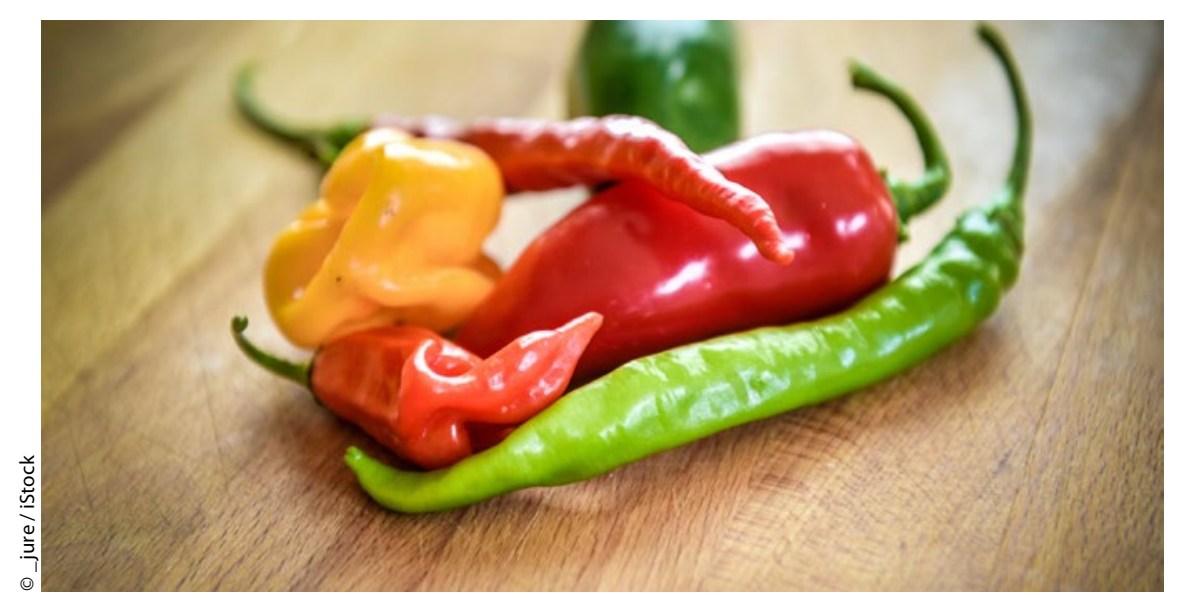

Das in manchen Paprika- und Chilischoten enthaltene Capsaicin hemmt hyperaktive Neuronen und damit den Schmerz auch bei Polyneuropahtien. 